\title{
TRATAMENTO RESTAURADOR ESTÉTICO DE DENTES COM ALTERAÇÃO DE COR - RELATO DE CASO CLÍNICO
}

\section{AESTHETIC RESTORATIVE TREATMENT OF TEETH WITH COLOR ALTERATION - CASE REPORT}

\author{
Veruska de João Malheiros Pfau', Priscila Gomes Tavares', \\ Márcio Grama Hoeppner ${ }^{{ }^{*}}$ \\ $1^{*}$ Autor para contato: Universidade Paranaense - UNIPAR - Departamento de Odontologia \\ - Umuarama, PR, Brasil; (44) 3621-2844; e-mail: hoeppner@uol.com.br \\ Recebido para publicação em 29/03/2006 \\ Aceito para publicação em 14/06/2006
}

\section{RESUMO}

O restabelecimento estético de dentes com alteração de cor representa uma das principais ações dos cirurgiões-dentistas à promoção de um sorriso representativo de saúde. O objetivo deste trabalho foi demonstrar, através do relato de caso clínico, uma proposta de tratamento para dentes escurecidos, com e sem vitalidade pulpar, pela técnica do clareamento dental externo e interno com peróxido de hidrogênio a 35\% (Opalescense Xtra - Ultradent), associado ao tratamento restaurador direto. Neste caso clínico, o paciente apresentava o elemento dental 11 escurecido, no qual foi realizado clareamento interno e externo, e também insatisfação com a coloração dos outros dentes superiores e inferiores, os quais se apresentavam vitais, e foram clareados externamente. Após o clareamento, o tratamento foi concluído com a substituição da restauração do dente 11 e reparo do 21 , onde se verificou que as técnicas utilizadas e o materiais escolhidos possibilitaram um ótimo resultado estético, com simplicidade de técnica e baixo custo, além de mínima ocorrência de sinais e sintomas.

Palavras-Chave: estética, clareamento dental.

\begin{abstract}
The aesthetic reestablishment of tooth with chromatic alteration represents one of principal dentist's conduct on promotion of a representative smile of health. The purpose of this work was to demonstrate, through a relate of a case, an option of treatment for teeth whit color alteration, with and without pulp's vitality, by the techniques of intra and extracoronal dental bleaching with 35\% hydrogen peroxide (Opalescense Xtra - Ultradent), associated to the direct treatment
\end{abstract}


restorative. In case report the patient had the dental element 11 (right upper central incisor) chromatic alteration, in which it was done intra and extra dental bleaching, the patient was too unsatisfied with the color of her other uppers and lowers teeth, which had vitality, and were extracoronally bleached. After the bleaching, the treatment was concluded with the substitution of dental element 11 restoration and repair of dental element 21(left upper central incisor), we could verify that the techniques utilized and the materials choices permitted an excellent aesthetic result, with simplicity of techniques and low cost, beyond of minimum happening of signals and sings.

Key words: aesthetic, dental bleaching

\section{Introdução}

Há tempos a estética passou a ser vista pelas pessoas como um fator importante para o bem estar social, emocional e até mesmo econômico. Dentre os fatores que influenciam o padrão de beleza identificamos a estética do sorriso, representada por dentes brancos e alinhados, não portadores de alteração de cor significativa, ou seja, livres de qualquer tipo de manchamento.

Mesmo que as condutas clínicas preventivas tenham resultado na redução do índice de cárie, atualmente, para contemplar os conceitos de beleza e saúde, cada vez mais as pessoas buscam nos tratamentos odontológicos uma opção para melhorar a aparência, em especial para solucionar problemas advindos da alteração de cor dos elementos dentais. Em conseqüência, novas técnicas e produtos foram propostos para suprir as necessidades do mercado. Sendo que alguns dos agentes clareadores comercializados priorizam a sua praticidade de utilização não exigindo a necessidade da prescrição e supervisão do cirurgião-dentista, em beneficio de um custo mais acessível.

Para Haywood e Heymann (1989), o uso de agentes oxidantes para contornar o problema estético decorrente da alteração de cor dos dentes, além de se mostrar um procedimento conservador, quando corretamente indicado, apresenta resultados satisfatórios, o que torna a técnica uma opção de tratamento. Agentes clareadores, a base de peróxido de hidrogênio e peróxido de carbamida, dependendo da concentração, podem ser empregados pela técnica caseira
(Night Guard Vital Bleaching) ou a nível ambulatorial (consultório).

De acordo com Pimenta (2002), para que o cirurgião-dentista possa obter maior índice de sucesso no tratamento clareador proposto, é de suma importância conhecer a etiologia da alteração de cor.

As causas mais freqüentes que podem levar ao escurecimento dental, são: a decomposição do tecido pulpar, hemorragia interna, trauma, uso de medicamentos, materiais restauradores e condições sistêmicas (fluorose, icterícia e eritroblastose fetal) (Venturine, 2001).

Para Cabral (2001), o clareamento dental é um tratamento que não deve ser sugerido pelo profissional, mas solicitado pelo paciente que, quando assim o fizer, deve ser alertado sobre os possíveis riscos.

Por razões estéticas, vale ressaltar que o clareamento dental com freqüência precede a realização de restaurações estéticas em resina composta nos dentes anteriores. Portanto, diante da associação de procedimentos em benefício da estética, este trabalho tem como objetivo apresentar uma proposta (opção) de tratamento para resolução estética de dentes com e sem vitalidade pulpar, portadores de alteração de cor.

\section{Relato do caso clínico}

O desejo em ter o incisivo central superior direito (dente 11) clareado, além de melhorar o padrão de coloração dos demais dentes anteriores, foi a razão do encaminhamento da paciente J.R., 22 anos de idade, à 
Clínica Odontológica da Universidade Paranaense UNIPAR - Umuarama. Após anamnese, avaliação clínica e radiográfica inicial, pôde-se diagnosticar que a alteração cromática do dente 11 era decorrente do tratamento endodôntico.

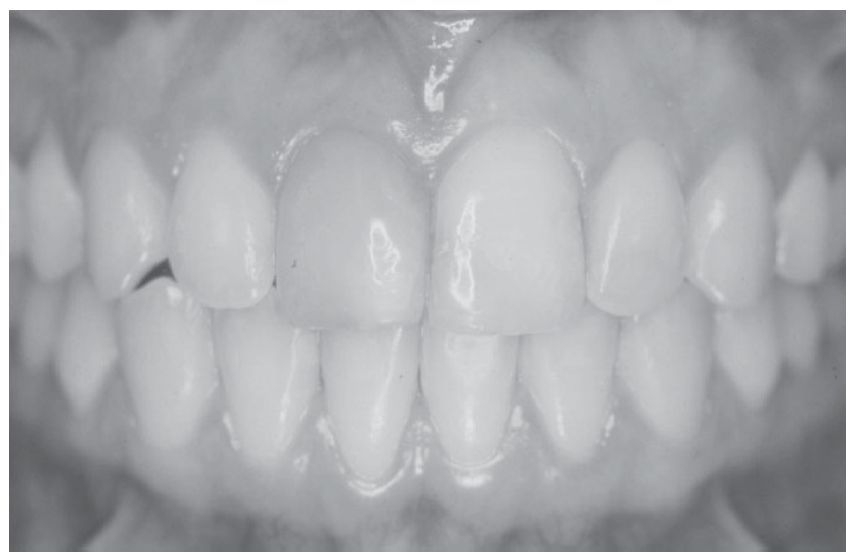

Figura 1 - Visão inicial

Frente às necessidades apresentadas pela paciente, o tratamento proposto foi a realização do clareamento interno no elemento 11 , sem vitalidade pulpar (tratado endodônticamente), e clareamento externo dos demais dentes ântero-superiores e inferiores, com peróxido de hidrogênio a 35\% (Opalescence Xtra - Ultradent), antes da substituição das restaurações estéticas em resina composta presentes nos dentes 11 e 21 .

$\mathrm{Na}$ primeira sessão, para a realização do clareamento interno do dente 11 , inicialmente foi removido o material restaurador do interior da câmara pulpar e, em relação ao limite gengival vestibular, $3 \mathrm{~mm}$ do material obturador na região cervical radicular. Todo o procedimento operatório foi, na seqüência, realizado com uma ponta diamantada esférica (1012 - KG Sorensen), em alta rotação, e broca esférica de haste longa (FGC4 - KG Sorensen), em baixa rotação.

Finalizada a abertura palatina, realizamos profilaxia dos dentes ântero-superiores com pasta de água e pedra-pomes, com auxílio de uma taça de borracha montada em baixa rotação. Em seguida, após a lavagem dos dentes, o tecido dentinário da câmara pulpar do dente 11 foi tratado com ácido poliacrílico a 11,5\% (condicionador de dentina Vidrion - SSWhite), lavado e seco. Em seguida, com cimento de ionômero de vidro modificado por resina composta (Fuji II LC, cor A2 - GC), foi confeccionado o tampão cervical (ou rolha cervical seladora), com o propósito de limitar a ação do agente clareador à região coronária, correspondente à câmara pulpar.

Precedendo a aplicação do agente clareador no dente 11 no interior da câmara pulpar e na superfície vestibular, para o clareamento do mesmo, foi aplicada resina composta fotopolimerizável para barreira gengival (OpalDan - Ultradente) para proteção do tecido gengival vestibulo-lingual.

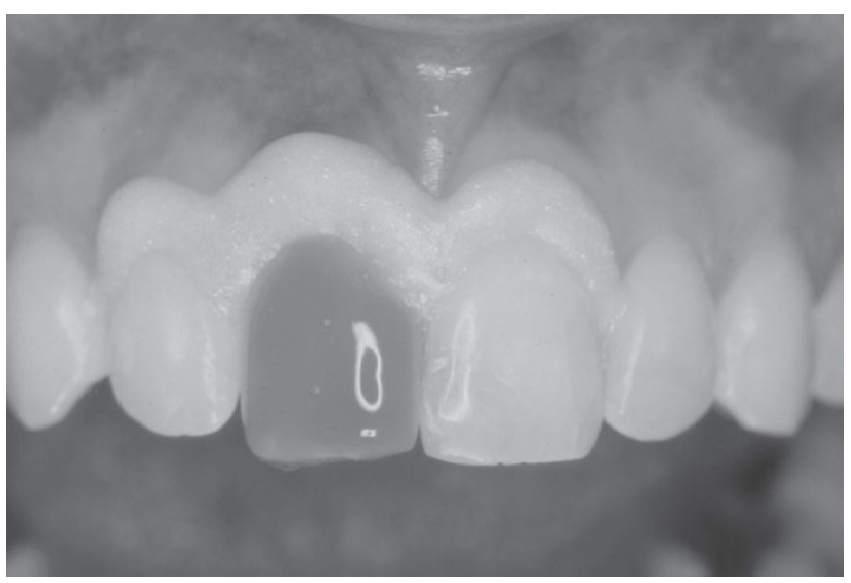

Figura 2 - Clareamento do dente 11: aplicação do agente clareador (Opalescente Xtra a 35\% - Ultradente).

A fotoativação dos produtos empregados (OpalDan - protetor gengival e Opalescence Xtra agente clareador) foi realizada com um aparelho de luz halógena (Optlight 600 - GNATUS), de acordo com as recomendações do fabricante. Ao término da sessão, a câmara pulpar do dente 11 foi preenchida com pasta de hidróxido de cálcio pró-análise (p.a.) e água destilada, e selada com cimento de ionômero de vidro (Vidrion R - SSWhite).

Passados sete dias, na segunda sessão, todos os dentes ântero-superiores (incluindo o dente 11) e ântero-inferiores foram submetidos à ação do mesmo agente clareador. Assim, após proteção do tecido gengival com Omcilon- A em Orabase (Bristol Myers Squibb Brasil S.A.) e realização do isolamento absoluto do campo operatório, realizamos profilaxia dos dentes a serem clareados com pasta de água e pedra-pomes, com auxílio de uma taça de borracha montada em baixa rotação. Em seguida, todos os dentes foram lavados e 
secos para receber a aplicação do gel de peróxido de hidrogênio a 35\% (Opalescence Xtra - Ultradent). O agente clareador foi aplicado sobre as faces vestibular e lingual de todos os dentes e fotoativado de acordo com as recomendações do fabricante, com um aparelho de luz halógena (Optlight 600 - GNATUS).

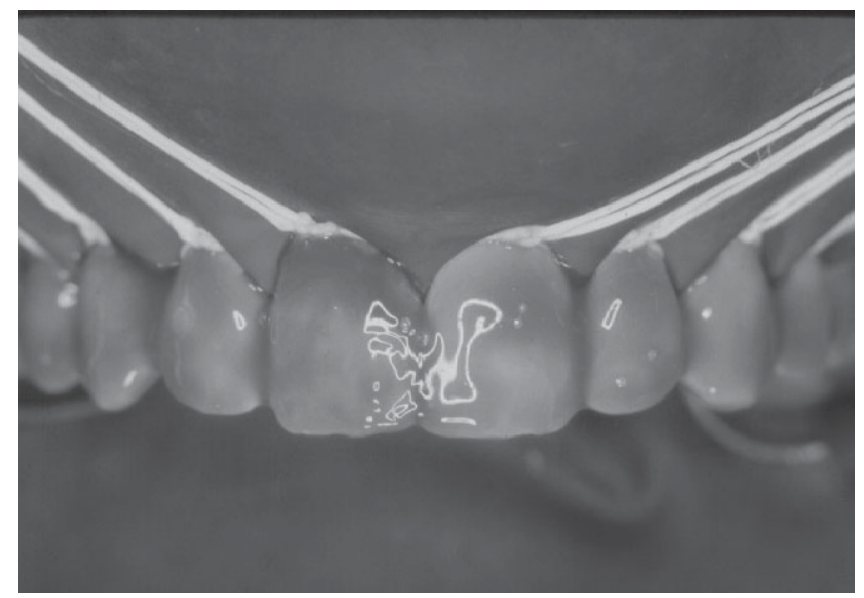

Figura 3 - Clareamento dos dentes ântero-superiores: aplicação do agente clareador (Opalescente Xtra a 35\% - Ultradente).

Passados 14 dias da segunda sessão, realizamos a substituição da restauração em resina composta do dente 11 e reparo da restauração do dente 21 . Dentro da técnica restauradora, considerando o grau de opacificação e a cor desejada, a seleção das resinas compostas utilizadas foi feita após profilaxia dos dentes ântero-superiores, sob isolamento relativo e num intervalo de tempo curto.

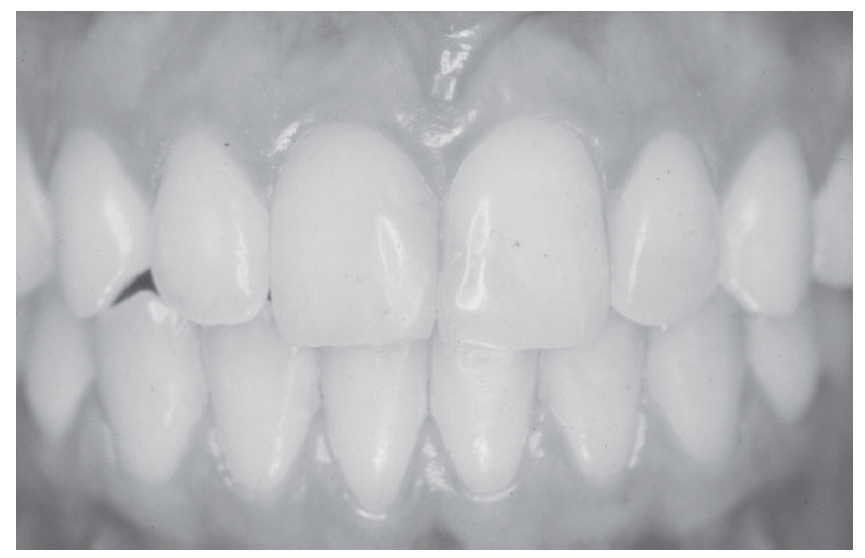

Figura 4 - Resultado estético após clareamento dos dentes ântero-superiores.
Para atender às exigências estéticas, selecionamos dois tipos de resina composta: uma resina composta híbrida, para preenchimento coronário e reconstrução palatina (Herculite XRV - Kerr) e uma resina composta microparticulada, para resolução estética vestibular(Durafill-Kulzer).

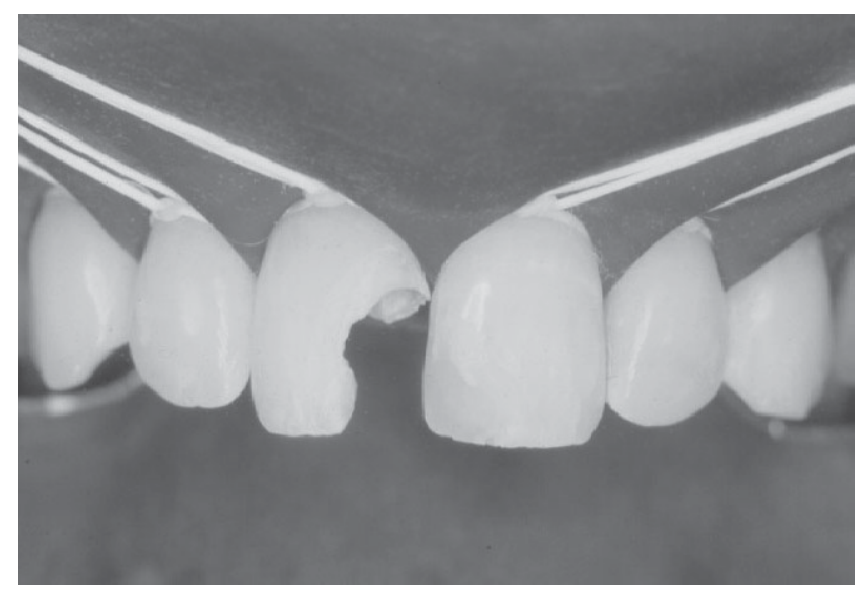

Figura 5 - Realização das restaurações estéticas dos incisivos centrais superiores 11 (sem vitalidade pulpar) e 21 (com vitalidade pulpar).

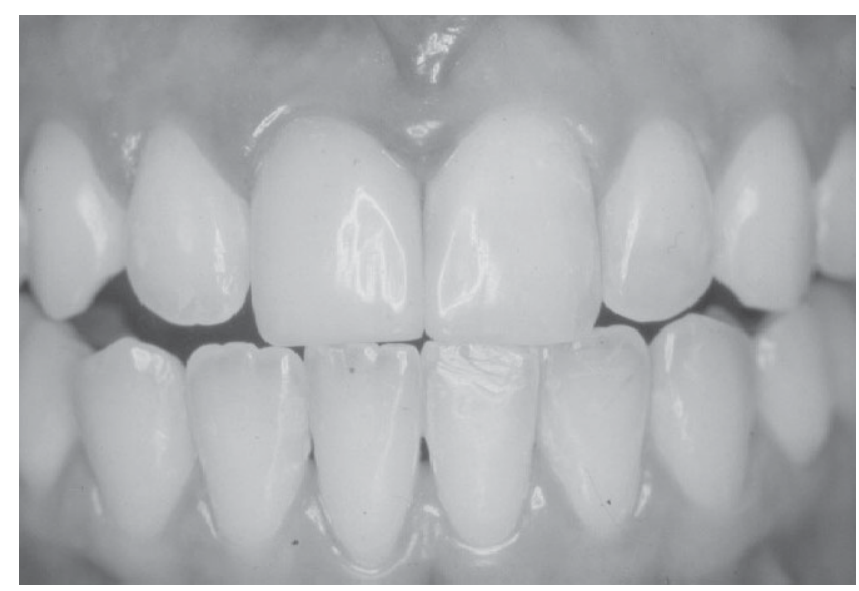

Figura 6 - Resultado estético final.

Uma vez concluída as restaurações, o acabamento para a remoção dos excessos cervicais e ajuste do contorno vestibular foi realizado com auxílio de lâmina de bisturi número 12, tiras e discos de lixa (SofLex Pop On XT - 3M). O polimento final foi realizado na sessão seguinte, com seqüencial de discos de lixa (Sof-Lex Pop On XT - 3M). Toda seqüência clínica está ilustrada nas Figuras 1 a 6. 


\section{Discussão}

Diante das exigências do mundo moderno, observamos que os pacientes, no desejo de terem dentes brancos, cada vez mais solicitam procedimento de clareamento (Junqueira, 2000). Conseqüentemente, o restabelecimento estético de dentes com alteração de cor representa um grande desafio aos cirurgiõesdentistas, em especial no que se refere ao prognóstico do tratamento realizado. Embora, quando comparado às técnicas restauradoras, o clareamento representa uma excelente opção em conseqüência dos resultados satisfatórios e preservação das estruturas dentais (Haywood e Heymann, 1989; Assis, 1999).

De acordo com Castello (2004), a utilização do peróxido de hidrogênio a $30 \%$ ativado por calor e luz de alta intensidade, desde 1918, forneceu alicerce para as técnicas de clareamento dental que atualmente são empregadas para o tratamento estético de dentes com ou sem vitalidade pulpar.

Entretanto, vale ressaltar que os agentes clareadores são veículos de radicais de oxigênio livres bastante instáveis que, em contato com os tecidos, promovem, por meio de oxi-redução dos pigmentos, o clareamento do elemento dental. O sucesso clínico da técnica somente é possível em conseqüência da permeabilidade do esmalte e dentina (Baratieri, 2001; Busato, 1997).

Para o clareamento de dentes tratados endodônticamente podemos fazer uso de diferentes agentes clareadores à base de peróxido, em diferentes concentrações e técnicas. Para Baratieri (2001), o peróxido de hidrogênio representa ser o material de escolha quando se fala em clareamento de dentes com ou sem vitalidade pulpar, devido à sua grande eficácia.

No que se refere ao clareamento de dentes sem vitalidade pulpar, tratados endodônticamente, a utilização do peróxido de hidrogênio na concentração entre 30 a $35 \%$ é o agente clareador mais utilizado, em associação ou não ao perborato de sódio (Benatti e Abe, 1971; Rotstein e Friedmans, 1991). Se avaliarmos a efetividade da técnica considerando o tempo necessário para o clareamento de dentes tratados endodônticamente, o peróxido de hidrogênio empregado como veículo em associação ao perborato de sódio é mais eficaz quando comparado à água destilada ou ao soro fisiológico Fraga (2000).
Quanto às desvantagens do procedimento de clareamento interno de dentes tratados endodônticamente, a possibilidade da reabsorção cervical externa representa um fator limitante da técnica (Montgomery, 1984; Cvek e Lindvall, 1985; Vieira, 1996).

Embora não se conheça a causa exata do fenômeno, a hipótese mais provável para justificar a ocorrência da reabsorção radicular externa seria a difusão do peróxido de hidrogênio através dos túbulos dentinários para a área do ligamento periodontal cervical, desnaturando a dentina e a caracterizando como uma estrutura imunologicamente diferente, induzindo a uma reação tecidual de resposta à presença de um corpo estranho. O processo de reabsorção inflamatória é semelhante ao que pode acontecer quando da luxação e reimplantação de dentes avulsionados (Harrington e Natkin, 1979; Madison e Walton, 1990). Contrário as essa afirmações, Rotstein (1991) afirmou não existir evidências científicas para comprovar que a penetração do material clareador possa provocar reação inflamatória seguida de reabsorção.

Mac Isaac (1994), após revisão da literatura, concluiu que em todos os casos de dentes tratados endodônticamente e submetidos ao procedimento de clareamento interno, descritos até o ano de 1994, havia algo que contribuía a ocorrência da reabsorção cervical externa. Em $100 \%$ dos casos em que foi diagnosticado reabsorção cervical, os dentes não tiveram seus condutos selados, ou seja, não foi confeccionado o tampão cervical; a técnica termocatalítica foi utilizada em $84 \%$ dos casos; e $72 \%$ dos pacientes com o problema possuíam história de trauma anterior ao clareamento.

Diante dos fatos, para diminuir a probabilidade da reabsorção radicular externa, justificamos a confecção do tampão cervical (ou rolha cervical seladora), com cimento de ionômero de vidro modificado com resina composta, com o propósito de promover o selamento dos túbulos dentinários nesta região. Também, concluído o clareamento interno do dente 11, preenchemos a câmara pulpar com pasta de hidróxido de cálcio p.a. e água destilada até a realização da restauração com resina composta. Esse procedimento foi realizado com o propósito de tornar o $\mathrm{pH}$ cervical alcalino, bem como dar tempo para a eliminação do oxigênio residual liberado pelo agente 
clareador, para não comprometer a polimerização da resina composta (Vieira, 1992; Montgomery, 1984; Baratieri, 1995; Assis, 1999; Martínez, 2003).

Outras condutas para reduzir os efeitos adversos do clareamento interno de dentes tratados endodônticamente é a associação de perborato de sódio e água (Vieira, 1992) ou a utilização de agentes clareadores utilizados na técnica preconizada por Haywood e Heymann: Nightguard Vital Bleaching (Friedmans, 1991).

Entretanto, escolhemos o peróxido de hidrogênio a $35 \%$ considerando o tempo clínico necessário à obtenção do resultado desejado, pois a ação do peróxido de hidrogênio é 3 a 6 vezes mais rápida quando comparado ao peróxido de carbamida (Monnerat, 1996).

Para o clareamento dos dentes ântero-superiores e inferiores com alteração de cor e vitalidade pulpar, selecionamos o mesmo agente clareador utlizado para o clareamento interno do dente 11 (Opalescence Xtra - Ultradente), aplicado aos dentes sob isolamento absoluto do campo operatório e fotoativado por um aparelho de luz halógena (Optilight 600 - GNATUS).

Para Cabral (2001), a não utilização de instrumentos aquecidos para potencializar a ação do agente clareador (técnica termocatalítica) também representa uma forma de evitar a ocorrência de reabsorção cervical. $\mathrm{O}$ autor afirmar ainda que, além da reabsorção cervical externa, em dentes com vitalidade pulpar, o uso da temperatura como forma de ativação da degradação do agente clareador pode causar sensibilidade pós-operatória.

A consistência do agente clareador selecionado foi outro fator considerado, pois, de acordo com Rodrigues (2004), o uso de agente clareador com maior quantidade de espessante limita a área de ação do mesmo sobre o dente a ser clareado e dificulta o seu escoamento para os tecidos, diminuindo, assim, os riscos de irritação gengival e reabsorção cervical.

Em relação à sensibilidade dentária, Barreiros (2002) afirma que não está relacionada com a exposição de tecido dentinário radicular ou a presença de lesão de cárie, mas é devido à fácil passagem da pequena molécula de peróxido de hidrogênio, através do esmalte e dentina, em direção ao tecido pulpar. Diz ainda, que aproximadamente dois terços dos pacientes em tratamento apresentam sensibilidade pós-operatória que, usualmente, dura de 1 a 4 dias.

Ressaltamos que o sucesso clínico alcançado pela técnica de clareamento dental está diretamente relacionada a presença de produtos ou substâncias químicas pigmentadas na cavidade bucal, que podem comprometer a longevidade do tratamento (Haywood, 1994).

Concluído o tratamento clareador, observamos que a mudança na coloração dos dentes clareados tornou evidente as restaurações em resina composta dos dentes 11 e 21. Entretanto, apesar da ansiedade estética do paciente e do profissional, esse procedimento não deve ser realizado imediatamente após o clareamento. Para Erhardt (2003) e Campos (2000), deve-se esperar um tempo entre duas a três semanas para que ocorra a liberação do peróxido de hidrogênio e seus subprodutos, que, quando presentes no interior dos túbulos dentinários, interferem no processo de polimerização dos materiais resinosos (Swift Jr, 1997), podendo, assim, comprometer a adesão à dentina.

\section{Conclusão}

Com base nos resultados estéticos obtidos após a realização do clareamento dos dentes com e sem vitalidade pulpar, e realização das restaurações em resina composta, podemos concluir que a conduta clínica proposta demonstrou: resultados clínicos satisfatórios, técnica acessível e mínima ocorrência de sinais e sintomas indesejáveis, desde que realizado com os devidos cuidados.

\section{REFERÊNCIAS}

1.ASSIS, B.R.PeALBUQUERQUE, R.C.Clareamento de dentes despolpados pela técnica Walking Bleach. Rev. do CROMG. v. 5, n.1, jan/abr, 1999.

2. BARATIERE, L.N.; JUNIOR, S.M.; ANDRADA, M.A.C. e VIEIRA, L.C.C. Clareamento dental. São Paulo: Santos/ Quintessence Books, p. 01-25, 1995.

3. BARATIERI, L.N.; JUNIOR, S.M.; ANDRADA, M.A.C.; VIEIRA, L.C.C.; RITTER, A.V. e CARDOSO,A.C. Odontologia 
Restauradora: Fundamentos e Possibilidades. São Paulo: Santos/Quintessence Books, p. 672-722, 2001.

4. BARREIROS, I.D.; ALVES, M.A.G.; DUTRA, P.M.M.; MENDONÇA, L.L. e FERREIRA, L.C.N. Tratamento clareador com peróxido de carbamida, Whiteness Super Endo (FGM) a 37\% em dentes não-vitais - Uma técnica. JBC, v. 1, n. 2, p. 140-145, abr/jun, 2002.

5. BENATTI, O. e ABE, A. Um método simplificado de branqueamento de dentes anteriores. RBO, n. 167, p. 20-26, 1971.

6. BUSATO, D.L.S.; BARBOSA, A.N.; BUENO, M. e BALDISSERA, R.A. Dentística: Restaurações em Dentes Anteriores. São Paulo/ Artes Médicas, p. 241-339,1997.

7. CABRAL, B.F. e OLIVEIRA, N.L. Uso combinado das técnicas de clareamento dental extracoronário e intracoronário. Rev.APCD, v. 55, n. 2, mar/abr, 2001.

8. CAMPOS, I. e PIMENTA, L.A.F. Substituição de restaurações após o clareamento dental caseiro. Rev. ABO Nac. v. 8, n. 2, abril/maio, 2000.

9. CASTEllo, R. R. e MONNERAT, A.F. Avaliação das alterações estruturais em esmalte submetido ao clareamento com peróxido de hidrogênio a 35\%. RBO, v. 61, n. 3 e 4, 2004.

10. CVEK, M. e LINDVALL, A.M. External root resorpition following of pulpless teeth with oxigen peroxide. Endod. Dent. Traumatol., p. 56-60, 1985.

11. ERHARDT, M.C.G.; SHINOHARA, M.S. e PIMENTA, L.A. Clareamento dental interno. RGO, 51 (1): 23-29, jan/fev/mar, 2003.

12. FRAGA, R.C.; SIQUIERA, J.F.J.; ASSAF, A.V. e TULCHINSKI, J. Comparação entre dois veículos para o perborato de sódio no clareamento de dentes despolpados. RBO, v. 57, n. 4, jul/ago, 2000.

13. HAYWOOD, V. B.; HEYMANN, H.O e ED, DDS. M. Nightguard vital bleaching. Quintessence Int., v. 20, n. 3, p. 173-6, 1989.

14. HAYWOOD, V.B.; LEONARD, R.H.; NELSON, C.F. e BRUNSON, W.D. Effectiveness, side effects and long term status of nightguard vital bleaching. J. Am. Dent. Assoc. 125:1219-26, 1994.

15. HARRINGTON, G.W. e NATKIN, E. External resorption associated with bleaching of pulpless teeth. J. Endod., v.5, p. 344-348, 1979.

16. JUNQUEIRA, J.C.; COLOMBO, C.E.D.; MARTINS, C.A.P.; TAVARES, P.G.; ARAÚJO, M.A.M. e VALERA, M.C. Efeito da técnica de clareamento, utilizando peróxido de carbamida a $35 \%$, sobre o esmalte dental - avaliação por microscopia de luz polarizada e microscopia eletrônica de varredura. JBC, n. 24, nov/dez, 2000.

17. MAC ISAAC, A.M. e HOEN, M.M. Journal, v. 60, n. 1, p. 57-64,jan, 1994.

18. MADISON, S. e WALTON, R. Cervical root resorption following bleaching of endodontically treated teeth. J. Endod., v. 16, p. $570-74,1990$.

19. MARTÍNEZ, D.P.; TEDESCO, A.D. e MIRANDA, M.S. Técnica mediata para clareamento de dentes desvitalizados: uma comparação clínica entre agentes clareadores - Relato de caso clínico. JBC, v.7, n.38, p. 138-142, 2003.

20. MONNERAT, A.F.; GARCIA, D.; SOUZA, L.; SANTOS, A.; VALIM, T.; LEITE, F. e ALONSO, L. Comparação clínica entre dois tipos de géis para clareamento vital. $\mathrm{RBO}, \mathrm{n} .4$, jul/ ago, 1996.

21. MONTGOMERY, S. External cervical resorpition after bleaching a pulpless tooth. Oral Surg., p. 203-06, 1984.

22. PIMENTA, L.A.F.; CASTRO,A.K.B.B. eERHARDT, M.C.G. Transformações estéticas do sorriso empregando procedimentos restauradores e não restauradores. $R B O$, v. 59, set/out, 2002.

23. RODRIGUES, J.A.; MONTAN, M.F. e MARCHI, G.M. Irritação Gengival após o Clareamento Dental. RGO, 52 (2): 111-114, abr/mai/jun, 2004.

24. ROTSTEIN, I. In vitro determination and quantification of $\mathbf{3 0} \%$ hydrogen peroxide penetration thorought dentin and cementum during bleaching. Oral Surg., Oral Med., Oral Pathol., v. 72, n. 5, p. 602-6, nov, 1991.

25. ROTSTEIN, I. e FRIEDMANS, S. pH variation among material used for intracoronal bleaching. J. Endod., v. 17, p. 376-9, 1991.

26. SWIFT JUNIOR, E.J. Restorative considerations with vital tooth bleaching. J. Am. Dent. Assoc., Chicago, v. 128, n. 4, p. 60s-64s, apr, 1997.

27. VENTURINI, M.H.D.; VENTURINI, M.D. e VENTURINI, M.S. Clareamento de dente: relato de caso clínico. Rev. Odontol. UNICID, v. 13, n. 1, p. 73-79, jan/abr, 2001.

28. VIEIRA, P.A.A. Reabsorção cervical pós-clareamento dentário - uma visão do estado atual. Marília, 1992. 55 p., Tese (Mestrado em Odontologia Restauradora) - Faculdade de Odontologia da Universidade de Marília.

29. VIEIRA, P.A.A. Avaliação da capacidade de selamento da região cervical de materiais usados na confecção do tampão cervical durante o clareamento dental intracoronário. Araraquara, 1996. 135 p., Tese (Doutorado em Dentística Restauradora) - Faculdade de Odontologia. Universidade Estadual Paulista. 